\title{
PROPHYLACTIC CPAP IN THE DELIVERY ROOM (DR) TO PREVENT TRANSIENT TACHYPNEA OF THE NEWBORN (TTN) AMONG INFANTS BORN VIA ELECTIVE CS (ECS), A PROSPECTIVE RCT
}

\author{
P. Srinivasan, P. Jain, M. Brandler, E. Lavery, E. Koenig, K. Williams, L. Cherian \\ Pediatrics, New York Hospital Queens, Flushing, NY, USA
}

Background: TTN from delayed clearance of fetal lung fluid (FLF) is common NICU admission among FT infants and require clinical management involving expensive resources, excessive and empiric antibiotics use. The increased risk of TTN among ECS born infants (CS in absence of labor) is a consequence of having excessive FLF with subsequent decreased release of counter regulatory hormones at delivery.

Aims: To determine whether prophylactic CPAP in the DR via T piece based Neopuff to neonates at risk for TTN, would decrease the incidence of TTN and NICU hospitalization.

Methods: IRB approved RCT, Inclusion: FTs born via ECS. Exclusion: presence of risk factors for sepsis, congenital malformations. Eligible infants randomized prior to delivery to intervention (I) group to receive CPAP of $5 \mathrm{~cm}$ \{administered to spontaneously breathing infants after a notable cry, for 10 minutes with $21 \%$ $\mathrm{O} 2$ \} or control (C) group managed with provision of standard care in the DR. Infant and maternal clinical data collected. All infants assessed for respiratory distress and any adverse complications.

Results: $\mathrm{n}=66\{\mathrm{C}=30, \mathrm{I}=36\}$, enrolled in the ongoing trial are included. No significant difference seen between $\mathrm{C}$ and I group including incidence of TTN or adverse complications (Air-leaks) from NCPAP. $7 \%$ of the mothers had history of previous babies with TTN.

Conclusions: In this interim analysis we did not see any adverse or beneficial effects of prophylactic CPAP in DR. Inclusion of data from all patients enrolled at time of presentation will permit more complete evaluation of the benefits in this patient cohort. 